TECHNICAL NOTE

\author{
H.-S. Liu \\ H.-W. Chung \\ C.-J. Juan \\ S.-Y. Tsai \\ C.-Y. Wang \\ C.-C. Chan \\ G.-S. Huang \\ M.-C. Chou \\ C.-S. Lee \\ C.-W. Ko \\ N.-Y. Cho \\ C.-Y. Chen
}

\section{Anomalous J-Modulation Effects on Amino Acids in Clinical 3T MR Spectroscopy}

SUMMARY: The signal-intensity loss from anomalous J-modulation effects due to chemical-shift displacement was investigated on amino acid groups (alanine, valine, leucine, and isoleucine) at 3T by using point-resolved ${ }^{1} \mathrm{H}$ spectroscopy in patients with brain abscess and phantom experiments. With a larger chemical shift between methyl and methine resonances, alanine shows a greater effect of signal-intensity cancellation compared with other amino acids around $0.9 \mathrm{ppm}$, resulting in noninverted doublets at a TE of $144 \mathrm{~ms}$.
$\mathbf{H}_{\mathrm{t}}$ igh-field MR imaging systems such as 3T strength aid in the detection of brain metabolites due to increased sensitivity. However, the clinical 3T system also increases the spatial mismatch of section-selective radio-frequency pulses due to a larger chemical shift difference at higher fields and reduced radio-frequency pulse bandwidths. ${ }^{1-4}$ The issue of limited radio-frequency bandwidths is especially prominent with the increasing popularity of parallel imaging with array coils, whose receive-only function demands radio-frequency pulse transmission from the generally inefficient body coil. One frequently encountered challenge of MR spectroscopy at highfield MR imaging systems is the anomalous J-modulation effect on the weakly coupled $\mathrm{AX}_{3}$ system, which could hamper identification of the ideally inverted lactate doublet at TE $=$ $144 \mathrm{~ms}^{1-4}$

Although clinical investigations of the anomalous J-modulation effects have centered on lactate ${ }^{1-4}$ with theoretic analysis described clearly in a previous article, ${ }^{5}$ the possible occurrence arising from other $\mathrm{AX}_{3}$ spin systems such as alanine or other amino acids, valine, leucine, and isoleucine, overlaid around $0.9 \mathrm{ppm}$ in brain abscess, ${ }^{6}$ to our knowledge, has never been reported. With similar coupling constants exerted on the methyl protons around $7 \mathrm{~Hz},{ }^{7}$ these metabolites containing methyl and methine groups also form a lactate-like coupled system with a TE-dependent phase evolution of the methyl resonance. In this study, we aimed to use both in vivo and in vitro experiments to show that the anomalous J-modulation

Received September 30, 2007; accepted after revision December 11.

From the Department of Electrical Engineering (H.-S.L., H.-W.C., S.-Y.T., C.-Y.W., M.-C.C.), National Taiwan University, Taipei, Taiwan; Department of Radiology (H.-S.L., C.-J.J., C.-Y.W., G.-S.H., C.-Y.C.), Tri-Service General Hospital, Taipei, Taiwan; Philips Medical Systems (C.-C.C.), Best, the Netherlands; Department of Chemistry (C.-S.L.), Tamkang University, Taipei, Taiwan; Department of Computer Science and Engineering (C.-W.K.), National Sun Yat-Sen University, Kaohsiung, Taiwan; and Institute of Biomedical Engineering (N.-Y.C.), National Yang-Ming University, Taipei, Taiwan.

Supported in part by National Science Council under grants NSC-95-2314-B-002-221-MY3 and NSC-95-2314-B-016-036-MY2

Please address correspondence to Cheng-Yu Chen, MD, Department of Radiology, TriService General Hospital, National Defense Medical Center, Taipei, Taiwan, Republic of China; e-mail: sandy0928@seed.net.tw

DOI 10.3174/ajnr.A1131 effect analogous to lactate is also present for alanine, but to a much lesser extent for other amino acids, on a clinical 3T MR imaging scanner.

\section{Description of Technique and Results}

We performed experiments on a 3T scanner (Philips Medical Systems, Best, the Netherlands) using the quadrature body (Q-body) coil for transmission and an 8-channel phased-array modified sensitivity encoding head coil for receiving, by using point-resolved ${ }^{1} \mathrm{H}$ spectroscopy sequence (PRESS). The Q-body coil had a maximum transmission B1 field of $13.5 \mu \mathrm{T}(\sim 590 \mathrm{~Hz})$. Single-voxel MR spectral data were obtained with $\mathrm{TR} / \mathrm{TE}=2000 / 40,144$, and/or $288 \mathrm{~ms}$ with 128 acquisitions (total scanning time, 4 minutes 56 seconds). Two patients with abscesses underwent MR spectroscopic examinations on this system. To investigate the anomalous J-modulation effect on alanine and amino acid groups, we performed in vitro experiments on four 1.5-L bottles of aqueous solution phantom containing $10 \mathrm{mmol} / \mathrm{L}$ of alanine, valine, leucine, and isoleucine, respectively. The phantom experiments were performed by using parameters identical to those of the patients, except that the voxel size was fixed at $25 \times 25 \times 25 \mathrm{~mm}^{3}$.

Figs 1 and 2 show the data from a 19-year-old man and a 42 -yearold woman with brain abscess, respectively. The doublet at $1.47 \mathrm{ppm}$ due to the presence of alanine was clearly visible in the $40-\mathrm{ms}$ and 288-ms TE MR spectra (Figs $1 B$ and $2 C$ ). However, for the first patient (Fig $1 C$ ), there was a positive peak centered on the same resonance which, if from alanine, is expected to exhibit an opposite sign of resonance (ie, inverted doublet) at $\mathrm{TE}=144 \mathrm{~ms}$. The spectrum acquired from the second patient with $\mathrm{TE}=144 \mathrm{~ms}($ Fig $2 B)$ also exhibited signal-intensity canceling, whereas the spectrum acquired with $\mathrm{TE}=$ $288 \mathrm{~ms}$ showed substantially larger and clearer alanine resonances than that with $\mathrm{TE}=144 \mathrm{~ms}$. The expected inverted doublet of alanine in the 144-ms TE spectra was hardly detectable. Similarly, both of these in vivo spectra also show no clearly identifiable phase inversion of lactate around $1.33 \mathrm{ppm}$ in the $\mathrm{TE}=144 \mathrm{~ms}$ spectra. In contrast, note that the amino acid groups near $0.9 \mathrm{ppm}$ for the 2 subjects showed clearly inverted spectral lines in the TE $=144 \mathrm{~ms}$ spectra without noticeable reduction in signal intensity. The in vivo observations are consistent with results from the phantom experiments, in which an incomplete inverted phase at TE $=144 \mathrm{~ms}$ was seen for the 

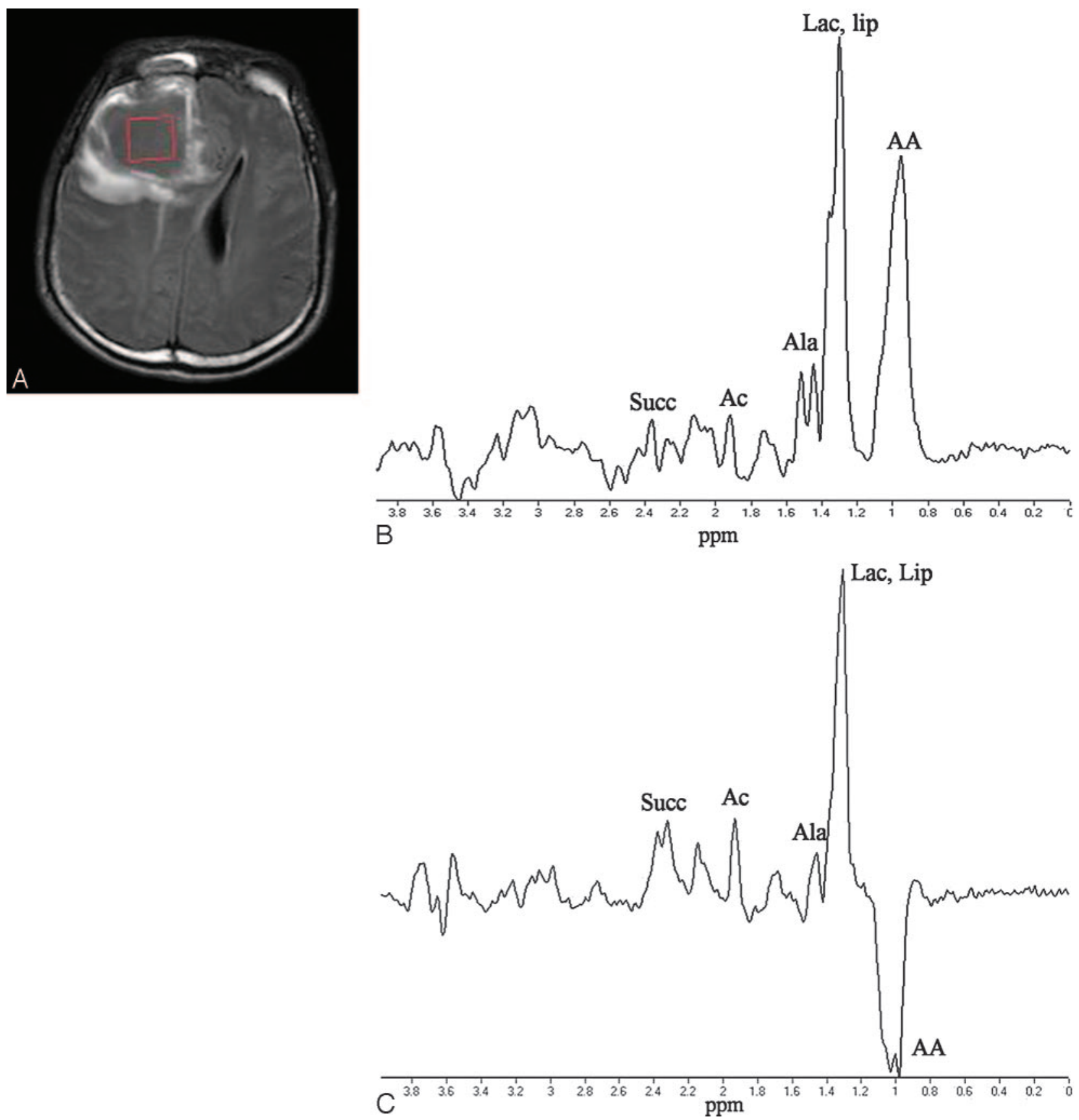

Fig 1. Transverse T2 fluid-attenuated inversion recovery MR image $(A)$ and single-voxel spectra obtained in a 19-year-old male patient with brain abscess by using PRESS localization at 3T with $\mathrm{TE}=40 \mathrm{~ms}(B)$ and TE $=144 \mathrm{~ms}(C)$. Succ indicates succinate; Ac, acetate; Ala, alanine; Lac, lactate; Lip, lipids; AA, amino acids.

methyl protons in alanine with reduced amplitude, as opposed to the strong signals in $\mathrm{TE}=40 \mathrm{~ms}$ and $\mathrm{TE}=288 \mathrm{~ms}$ spectra (Fig 3), whereas valine (Fig 4), leucine, and isoleucine (not shown) showed clear inverted peaks at $\mathrm{TE}=144 \mathrm{~ms}$ for the methyl protons with an amplitude comparable with that in $\mathrm{TE}=40 \mathrm{~ms}$ and $\mathrm{TE}=288 \mathrm{~ms}$ spectra.

\section{Discussion}

In this study, we demonstrated that the anomalous J-modulation effect analogous to lactate is also present for alanine on a clinical 3T scanner. Our inference suggests that the degree of reduction in sensitivity for alanine detection at TE $=144 \mathrm{~ms}$ is similar to that of lactate detection, due to a similar chemical shift difference between the methyl and the methine group protons $(2.78 \mathrm{ppm}$ or $36 \mathrm{~Hz}$ at $3 \mathrm{~T}$ for lactate, and $2.31 \mathrm{ppm}$ or $296 \mathrm{~Hz}$ at $3 \mathrm{~T}$ for alanine, respectively). ${ }^{7}$ On our system in which the refocusing radio-frequency bandwidth is $590 \mathrm{~Hz}$, $>50 \%$ of the methine protons within the section profile may thus exceed the spectral width without experiencing radiofrequency refocusing for each of the two $180^{\circ}$ pulses. ${ }^{3,5}$ Depending on whether the methine protons within the excitation volume experience the second and/or the third radio-frequency pulses in the PRESS sequence, the excitation volume thus is divided into 4 partial volumes, each with a different phase evolution that leads to destructive interference and consequent signal-intensity loss particularly prominent at $\mathrm{TE}=$ $144 \mathrm{~ms}(1-3,5)$. 

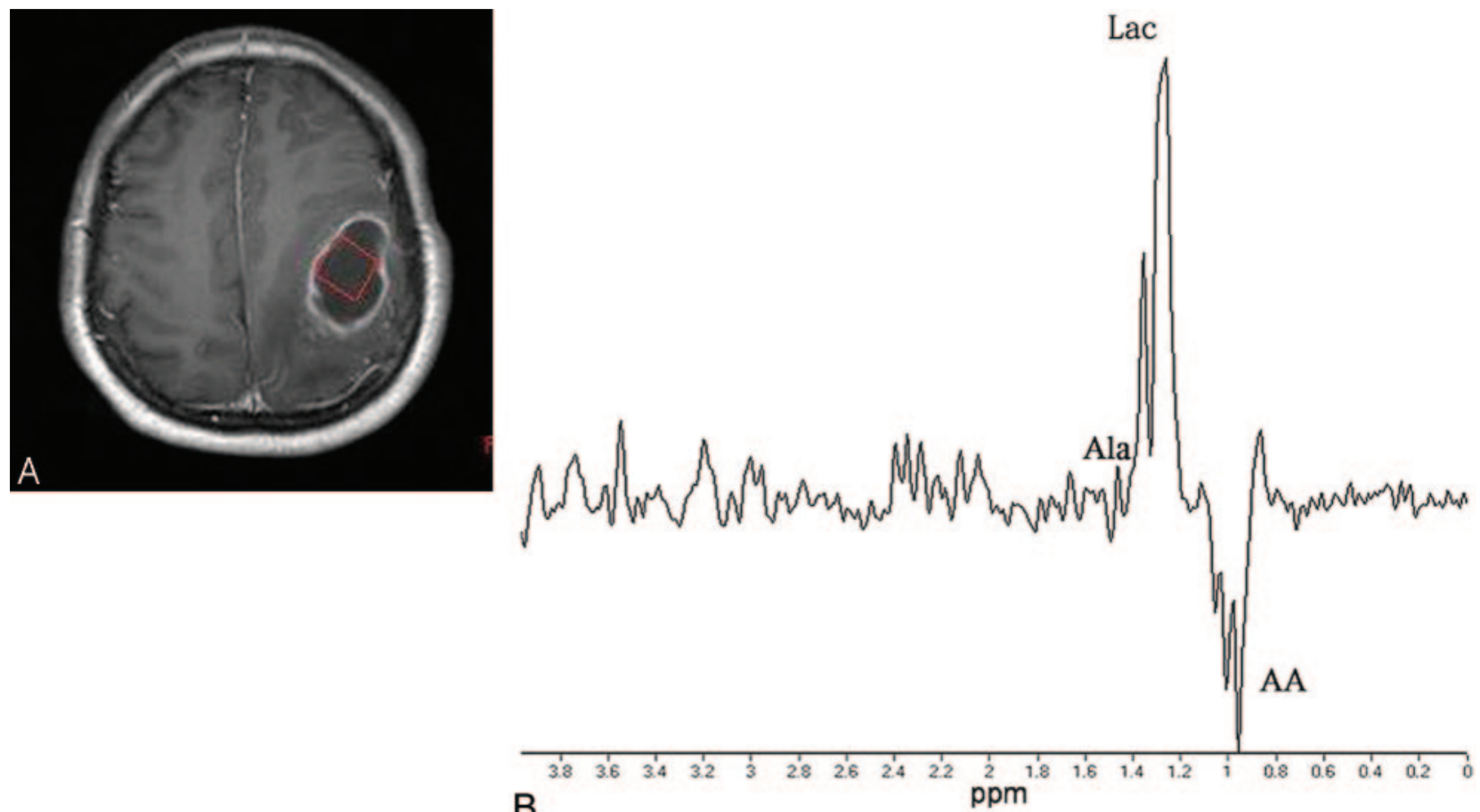

B

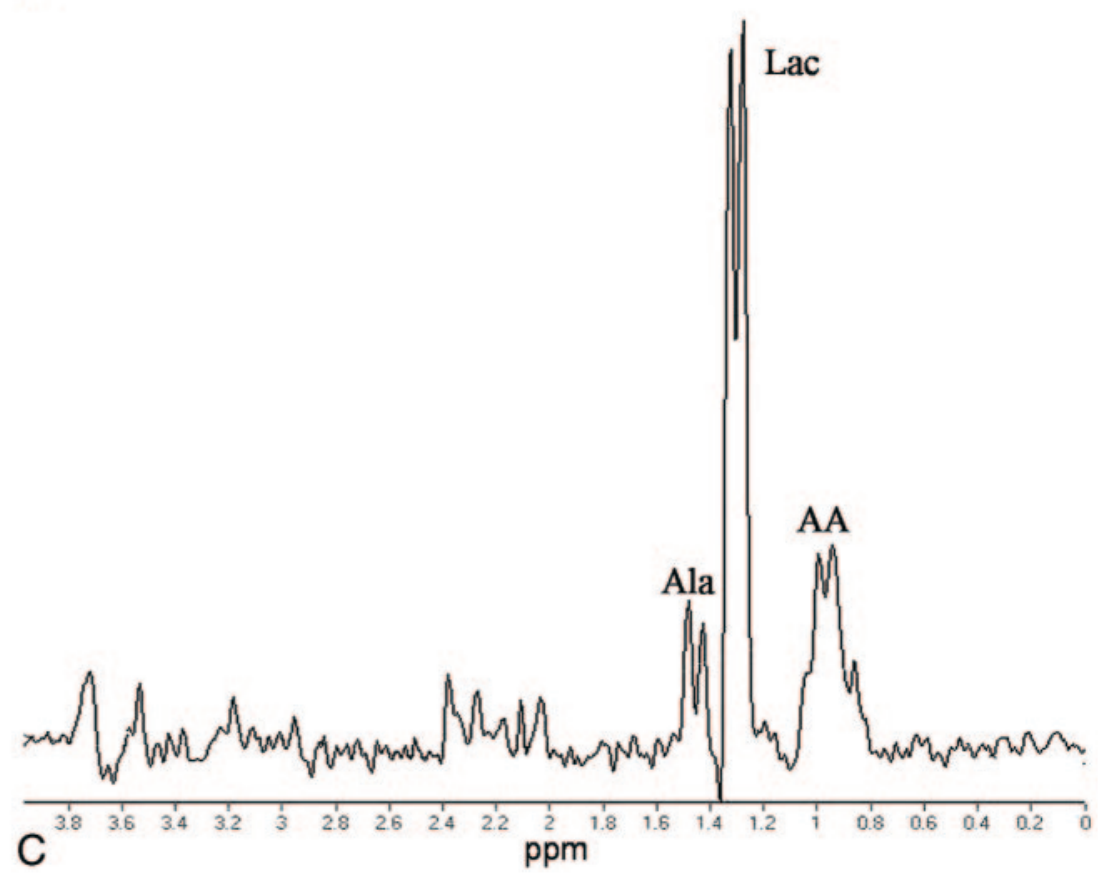

Fig 2. Transverse postcontrast T1-weighted MR image $(A)$ and single-voxel proton MR spectra obtained in a 42-year-old female patient with brain abscess by using PRESS localization at $3 \mathrm{~T}$ with $\mathrm{TE}=144 \mathrm{~ms}(B)$ and $\mathrm{TE}=288 \mathrm{~ms}(C)$. Ala, indicates alanine; Lac, lactate; $\mathrm{AA}$, amino acids.

In contrast to the situation of alanine, the chemical shift difference between the methyl and methine resonances is approximately $1.23 \mathrm{ppm}(157 \mathrm{~Hz}$ at $3 \mathrm{~T})$ for valine and $0.70 \mathrm{ppm}$ ( $89 \mathrm{~Hz}$ at $3 \mathrm{~T}$ ) for leucine, respectively, ${ }^{7}$ more than a factor of 2 smaller than that found for lactate. Consequently, the signalintensity cancellation effect is anticipated to be substantially weaker than that for lactate and alanine. In other words, spectra acquired with $\mathrm{TE}=144 \mathrm{~ms}$ may not show clearly noticeable anomalous J-modulation for the amino acids near the 0.90 -ppm region in the spectra, as shown in both our in vivo and in vitro data.
Alanine is potentially a pathologic marker of abscess resulting from bacterial infections of all kinds. ${ }^{6}$ It has also been observed following ischemia ${ }^{8}$ or in meningioma. ${ }^{9}$ Although alanine is not regarded as the most important biomarker for the differential diagnosis between tumor and abscess, ${ }^{8}$ a number of animal studies have addressed its potential role in monitoring metabolic alterations following brain injuries. ${ }^{10,11}$ Furthermore, the concentration of alanine in the brain, if detectable by in vivo MR spectroscopy, is usually small compared with that of lactate in the presence of prominent anaerobic glycolysis. This means that with destructive signal-inten- 


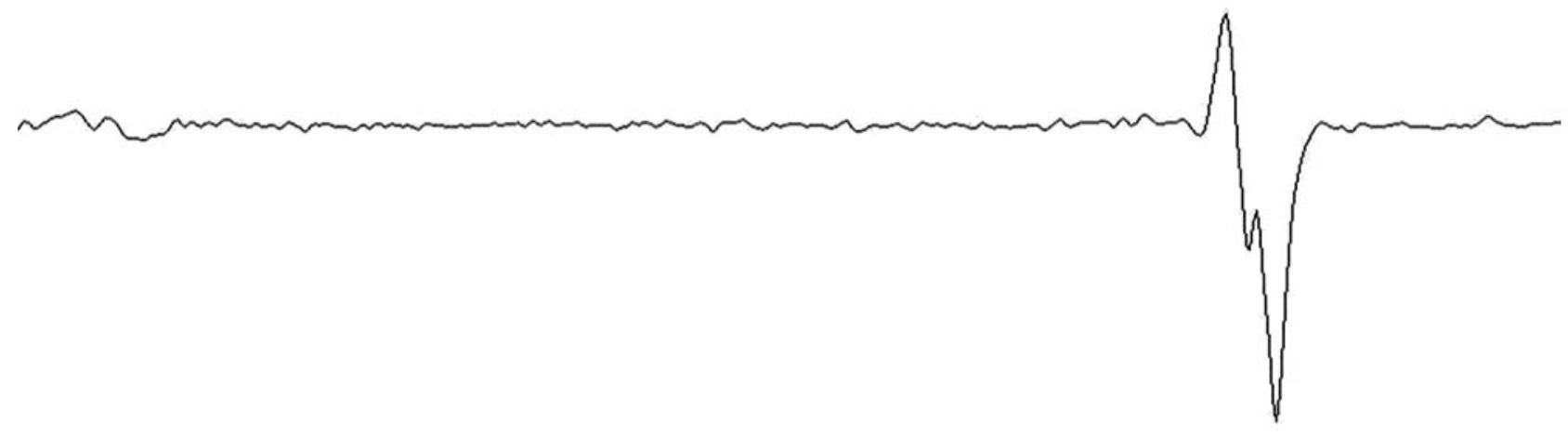

\begin{tabular}{|c|c|c|c|c|c|c|c|c|c|c|c|c|c|c|}
\hline $\mathrm{A}^{3.8}$ & 3.6 & 3.4 & 3.2 & 3 & 2.8 & 2.6 & ppm & 2.2 & 2 & 1.8 & 1.6 & 1.4 & 1.2 & 1 \\
\hline
\end{tabular}

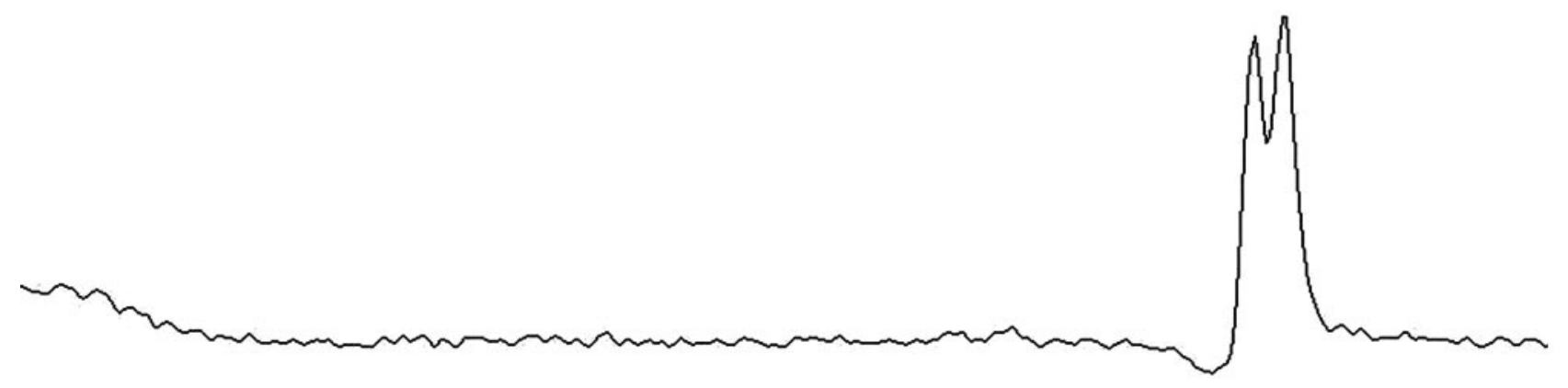

\begin{tabular}{|c|c|c|c|c|c|c|c|c|c|c|c|c|c|c|}
\hline$B^{3.8}$ & 3.6 & 3.4 & 3.2 & 3 & 2.8 & 2.6 & $\begin{array}{r}2.4 \\
\mathrm{pp}\end{array}$ & 2.2 & 2 & 1.8 & 1.6 & 1.4 & 1.2 & 1 \\
\hline
\end{tabular}

Fig 3. Spectra from a phantom that contained $10 \mathrm{mmol} / \mathrm{L}$ of alanine to illustrate the anomalous $\mathrm{J}$-modulation effect of the PRESS sequence at TE $=144 \mathrm{~ms}$ obtained from a $3 \mathrm{~T}$ scanner, with the identical setting in the receiver gain at TE $=144(A)$ and TE $=288 \mathrm{~ms}(B)$. Note at $1.47 \mathrm{ppm}$ the incompletely inverted phase and reduced amplitude of the methyl doublet in the $\mathrm{TE}=144 \mathrm{~ms}$ spectrum, evidence of the presence of an anomalous J-modulation effect.

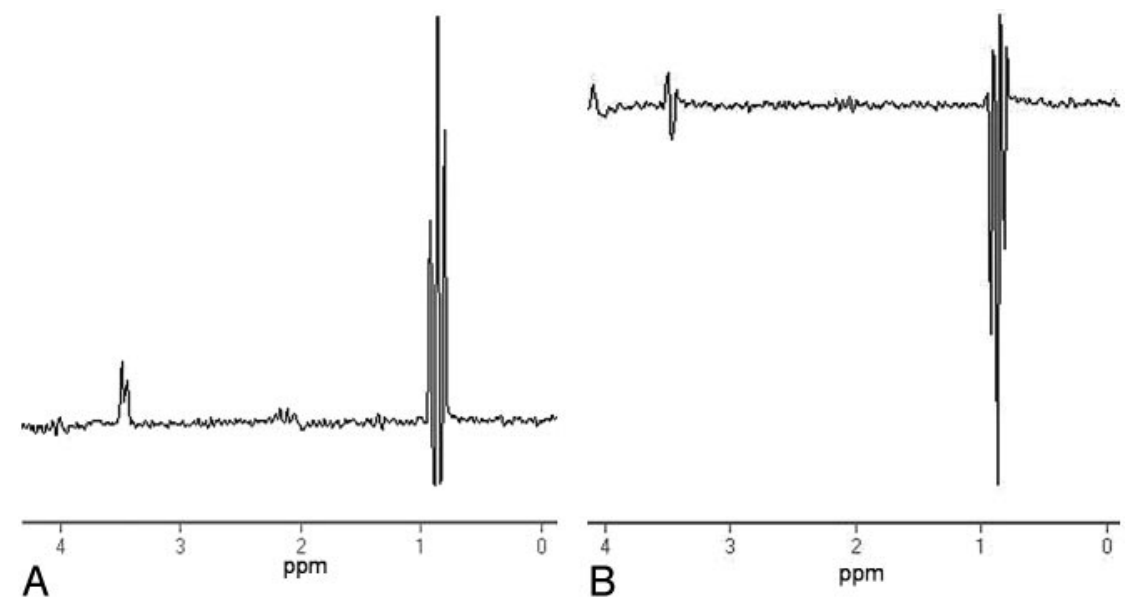

Fig 4. Spectra from a phantom that contained only valine at $\mathrm{TE}=40 \mathrm{~ms}(A)$ and $\mathrm{TE}=144 \mathrm{~ms}(B)$. The complex doublets between 0.90 and 1.06 ppm show a clearly inverted phase at $\mathrm{TE}=144 \mathrm{~ms}$. 
sity loss due to anomalous J-modulation effects, the identification of alanine at TE $=144 \mathrm{~ms}$ by using PRESS is even more prone to error than the situation expected for lactate. Special care in spectral interpretation related to alanine is therefore recommended at high-field systems such as 3T.

\section{References}

1. Lange T, Dydak U, Roberts TPL, et al. Pitfalls in lactate measurements at 3T. AJNR Am J Neuroradiol 2006;27:895-901

2. Edden RA, Schar M, Hillis AE, et al. Optimized detection of lactate at high fields using inner volume saturation. Magn Reson Med 2006;56:912-17

3. Kelley DA, Wald LL, Star-Lack JM. Lactate detection at 3T: compensating J coupling effects with BASING. J Magn Reson Imaging 1999;9:732-37

4. Klomp DW, van der Graaf M, Willemsen MA, et al. Transmit/receive headcoil for optimal $1 \mathrm{H}$ MR spectroscopy of the brain in paediatric patients at $3 \mathrm{~T}$. MAGMA 2004;17:1-4. Epub 2004 May 24
5. Yablonskiy DA, Neil JJ, Raichle ME, et al. Homonuclear J coupling effects in volume localized NMR spectroscopy: pitfalls and solutions. Magn Reson Med 1998;39:169-78

6. Garg M, Gupta RK, Husain M, et al. Brain abscesses: etiologic categorization with in vivo proton MR spectroscopy. Radiology 2004;230:519-27

7. Govindaraju V, Yang K, Maudsley AA. Proton NMR chemical shifts and coupling constants for brain metabolites. NMR Biomed 2000;13:129-53

8. Grand S, Passaro G, Ziegler A, et al. Necrotic tumor versus brain abscess: importance of amino acids detected at $1 \mathrm{H}$ MR spectroscopy-initial results. $R a$ diology 1999;213:785-93

9. Demir MK, Iplikcioglu AC, Dincer A, et al. Single voxel proton MR spectroscopy findings of typical and atypical intracranial meningiomas. Eur J Radiol 2006;60:48-55

10. Pascual JM, Solivera J, Prieto R, et al. Time course of early metabolic changes following diffuse traumatic brain injury in rats as detected by (1)H NMR spectroscopy. J Neurotrauma 2007;24:944-59

11. Melo TM, Nehlig A, Sonnewald U. Metabolism is normal in astrocytes in chronically epileptic rats: a (13)C NMR study of neuronal-glial interactions in a model of temporal lobe epilepsy. J Cereb Blood Flow Metab 2005;25:1254-64 